\title{
Effects of immersion in solution of an experimental toothpaste containing S-PRG filler on like-remineralizing ability of etched enamel
}

\author{
Masahiro IIJIMA ${ }^{1}$, Shuichi ITO², Susumu NAKAGAKIㄴ, Naohisa KOHDA', Takeshi MUGURUMA ${ }^{1}$, Takashi SAITO² \\ and Itaru MIZOGUCHI ${ }^{1}$ \\ ${ }^{1}$ Division of Orthodontics and Dentofacial Orthopedics, Department of Oral Growth and Development, School of Dentistry, Health Sciences University \\ of Hokkaido, 1757 Kanazawa, Ishikari-Tobetsu, Hokkaido 061-0293, Japan \\ ${ }^{2}$ Division of Clinical Cariology and Endodontology, Department of Oral Rehabilitation, School of Dentistry, Health Sciences University of Hokkaido, \\ 1757 Kanazawa, Ishikari-Tobetsu, Hokkaido 061-0293, Japan \\ Corresponding author, Masahiro IIJIMA; E-mail: iijima@hoku-iryo-u.ac.jp
}

This study investigated the like-remineralizing ability of experimental toothpaste containing surface reaction-type pre-reacted glassionomer (S-PRG) filler on etched enamel. Human enamel blocks were etched with 35\% phosphoric acid and immersed in 5-mL distilled water, fourfold diluted solution of NaF-containing toothpaste, or S-PRG filler-containing experimental toothpaste. Nanoindentation testing was carried out during immersion and the enamel surfaces were observed by scanning electron microscopy. Elemental analysis of the ions in each solution was performed using inductively coupled plasma atomic emission spectroscopy and fluoride electrode. After 1 month of immersion, the hardness and elastic modulus of the specimen immersed in S-PRG filler-containing toothpaste showed significantly greater values than those of the specimen immersed in NaF-containing toothpaste. Considerable amounts of $\mathrm{Al}, \mathrm{B}, \mathrm{Na}, \mathrm{Si}$, Sr, F ions were detected in the solution of S-PRG filler-containing toothpaste. Experimental S-PRG filler-containing toothpaste may enhance the like-remineralizing ability of etched enamel surfaces due to its ion-releasing ability.

Keywords: Remineralization, Enamel, Nanoindentation, Ion-releasing, S-PRG

\section{INTRODUCTION}

An acid-etching technique was introduced in the 1950s by Buonocore ${ }^{1}$. Since the direct bonding of orthodontic brackets to enamel was introduced by Newman ${ }^{2}$ in the mid 1960s, acid-etching of enamel surfaces for bracket bonding procedures has been accepted in clinical orthodontics ${ }^{3}$. Also, pit-and-fissure sealant using resinbased materials that require an acid-etching process has been accepted to prevent occlusal caries in pediatric dentistry ${ }^{4}$. A previous in vitro study ${ }^{5}$ showed that the mechanical properties of the enamel surface region were decreased by etching-based bracket bonding, and irreversible alteration of the enamel might increase the risk of enamel micro cracks during debonding procedures. Therefore, the enhancement of remineralization in etched enamel regions is ideally required after bracket bonding and resin-based sealant application.

To enhance the remineralization of etched enamel surfaces, increased calcium or fluoride concentrations in oral fluids would seem reasonable ${ }^{6,7)}$. Because fluoride plays an important role in the prevention of demineralization, fluoride-containing products, such as toothpaste, mouth rinse, fluoride-releasing adhesives and sealants, have been widely introduced in dentistry ${ }^{8-10)}$. Surface pre-reacted glass-ionomer (S-PRG) technology, which forms a stable glass-ionomer phase in fillers by pre-reacting acid-reactive glass-containing fluoride with polycarboxylic acid in the presence of

Color figures can be viewed in the online issue, which is available at J-STAGE.

Received Aug 19, 2013: Accepted Mar 18, 2014

doi:10.4012/dmj.2013-224 JOI JST.JSTAGE/dmj/2013-224 water, was introduced recently ${ }^{11,12)}$, and the S-PRG fillers have the ability to release Al, B, F, Na, Si, and $\mathrm{Sr}$ ions. Si and $\mathrm{F}$ ions are known to be strong inducers of remineralization of the dentin matrix ${ }^{13)}$. Sr and $\mathrm{F}$ ions also improve the acid resistance of teeth by acting on hydroxyapatite to convert it to strontiumapatite ${ }^{14,15)}$ and fluoroapatite, respectively ${ }^{14,16,17)}$.

The purpose of this in vitro study was to investigate the like-remineralizing ability of a new experimental toothpaste containing S-PRG filler on a human etched enamel surface. Conventional fluoride-containing toothpaste was used as a reference for comparative purposes. We hypothesized that application of S-PRG filler-containing toothpaste would result in likeremineralizing ability equal to that of fluoride-containing toothpaste.

\section{MATERIALS AND METHODS}

\begin{abstract}
Materials
Eighteen human noncarious premolars, obtained by extraction from patients, who were undergoing orthodontic treatment, were subjected to nanoindentation tests of the buccal enamel surface. Surfaces before and after nanoindentation testing were observed using a scanning electron microscope (SEM). Figure 1 shows a schematic illustration of the specimen preparation sequence for the in vitro like-mineralization of etched enamel surfaces. All premolars were cut with a slow-speed water-cooled diamond saw (Isomet, Buehler, Lake Bluff, IL) so that they were divided into
\end{abstract}




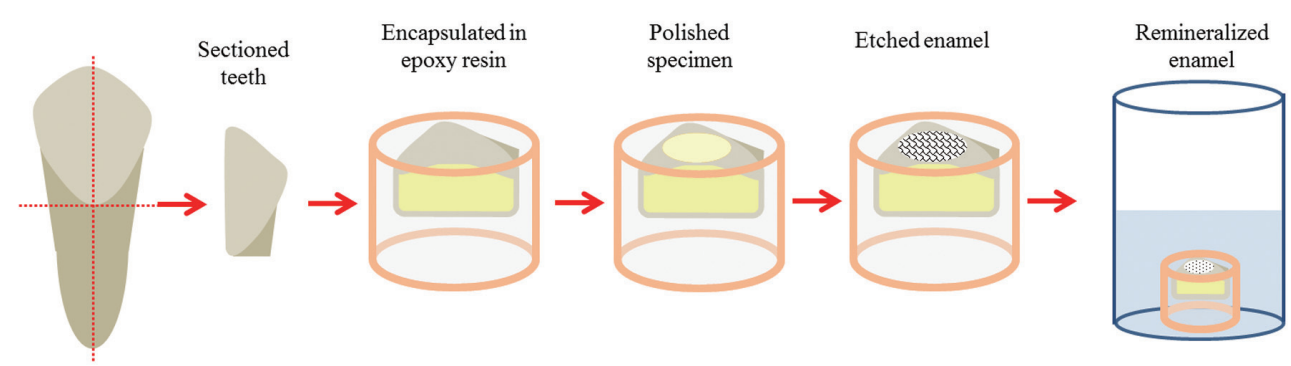

Fig. 1 Schematic illustration of the specimen preparation sequence for in vitro mineralization of an etched enamel surface.

mesial and distal halves after their roots were cut off; the sectioned specimens were then encapsulated in epoxy resin (Epofix, Struers, Copenhagen, Denmark). After $24 \mathrm{~h}$, the specimens were ground slightly (600-grit sandpaper) and polished using diamond suspensions. This polishing procedure removed approximately 200 $\mu \mathrm{m}$ of the tooth surface; polished-surface enamels with an approximate area of $4 \times 4 \mathrm{~mm}$ were finally obtained.

S-PRG filler was used to create an experimental toothpaste, which contained hydrated silica, carboxy methylcellulose, sodium, glycerol, sorbitol, sodium lauryl sulfate, flavor and SPR-G filler (5 and $30 \mathrm{wt} \%$ ). The technique of S-PRG filler manufacture is shown in a previous publication that described the effects of S-PRG fillers on mineral induction by phosphoproteins ${ }^{12)}$.

Etching by phosphoric acid and remineralization by immersion in each solution

The specimens (polished buccal enamel surfaces with approximate areas of $4 \times 4 \mathrm{~mm}$ ) were etched with $35 \%$ phosphoric acid gel (Transbond XT Etching Gel, 3M Unitek, Monrovia, CA) for $15 \mathrm{~s}$, washed for $20 \mathrm{~s}$, and dried with an air stream (Fig. 1). The etched specimens were then immersed in $5 \mathrm{~mL}$ of distilled water in a plastic vial, fourfold-diluted solution of $\mathrm{NaF}$ (950 ppm)containing toothpaste (Check-Up Standard, Lion Dental Products), or fourfold-diluted solution of S-PRG fillercontaining (5 or $30 \mathrm{wt} \%$ ) experimental toothpaste at $37^{\circ} \mathrm{C}$ for 3 months $(n=7)$.

\section{Measurements of hardness and elastic modulus by} nanoindentation tests

Nanoindentation testing of buccal enamel surfaces, involving production of five indentations in different regions of each specimen, was carried out at $28^{\circ} \mathrm{C}$ (ENT1100a, ELIONIX, Tokyo, Japan) using a $10 \mathrm{mN}$ load before etching, after etching, and during immersion periods ( 1 or 3 days, 1 week, 1 or 3 months). Linear extrapolation methods (ISO Standard 14577) were used for the unloading curve between $95 \%$ and $70 \%$ of the maximum test force to calculate the elastic modulus ${ }^{18}$. The hardness and elastic modulus of the buccal enamel surfaces were calculated using the software provided with the nanoindentation apparatus.
SEM observations and energy dispersive spectroscopy (EDS)

After nanoindentation testing of the buccal enamel surface (after immersion for 3 months), representative specimens from each group were observed by SEM (SSX550, Shimadzu, Kyoto, Japan). The specimens were sputter-coated with gold (SC-701 AT, Sanyu Electron, Tokyo, Japan) and examined using an SEM operating at $15 \mathrm{kV}$. Surfaces of non-etched and etched specimens were observed for comparison. The composition of the specimen surfaces (Ca, P, Sr, $\mathrm{Al}, \mathrm{Na}, \mathrm{F}$ ) for representative specimens was determined by EDS analysis with a working distance of $15 \mathrm{~mm}$. Five locations for each specimen were chosen for analysis.

\section{Analysis of released ions}

Fourfold-diluted NaF-containing toothpaste and S-PRG filler-containing toothpastes (toothpaste: $12 \mathrm{~g}$; distilled water: $36 \mathrm{~g}$ ) were prepared. After $24 \mathrm{~h}$, elemental analysis of the ions in supernatant liquid of each solution after centrifugal separation was performed using ICP-AES (inductively coupled plasma atomic emission spectroscopy) (ICPS-8000, Shimadzu, Kyoto, Japan) after preparing calibration curves with ionic concentrations from 0 to $20 \mathrm{ppm}$ corresponding to each element $(n=4)$. The ionic fluoride concentration in the solution was also quantified using fluoride ion-selective electrodes (Model 720A, Orion Research, Beverly, MA, USA) connected to an ion analyzer (F-53, Horiba). To stabilize the $\mathrm{pH}, 0.1 \mathrm{~mL}$ of TISAB III (Orion Research) was added to the solution. The electrodes were calibrated with a series of standard fluoride solutions with ionic concentrations from 0.02 to $20 \mathrm{ppm}$ of fluoride. A calibration graph was prepared and used to calculate the concentration of fluoride in each sample solution.

\section{Statistical analysis}

The experimental results were analyzed using PASW Statistics software (ver. 18.0J for Windows, IBM, Armonk, NY). The mean hardness and elastic modulus values, along with the standard deviations, for the five groups were compared by one-way ANOVA, followed by Tukey's test. For all statistical tests, significance was predetermined at $p<0.05$. 


\section{RESULTS}

Figure 2 shows mean values of hardness and elastic modulus of the buccal enamel surface before etching, after etching, and during the 3-month immersion period. The results of the statistical comparisons of the four specimen groups are summarized in Tables 1 and 2. There was no significant difference in the hardness or elastic modulus in any group before etching and immediately after etching. Although the hardness and elastic modulus values of specimens immersed in distilled water after etching were constant during the 3-month immersion, the values of the other three specimens increased gradually. After 1 and 3 months' immersion, the hardness and elastic modulus of the specimen immersed in S-PRG filler-containing toothpaste showed significantly greater values than that of the specimen immersed in $\mathrm{NaF}$ (950 ppm)containing toothpaste.

The polished buccal enamel surface after etching with 35\% phosphoric acid for $15 \mathrm{~s}$ showed a very porous surface and numerous enamel prisms could be observed, reflecting a typical honeycomb pattern (Fig. 3). After immersion for 3 months in the solutions, the honeycomb-like structure caused by acid etching was partly filled by a like-remineralized layer. In particular, S-PRG filler-containing toothpaste (30\%) showed progressive micro-particle deposition. The elements detected by EDS on the surface of the enamel are summarized in Table 3. Similar amounts of calcium, phosphate, fluorine and strontium were detected in all specimens.

Table 4 summarizes the amounts of ions detected in each solution after $24 \mathrm{~h}$. Several ions, such as Al, B, $\mathrm{Na}, \mathrm{Si}, \mathrm{Sr}, \mathrm{F}$, were detected in the solutions containing NaF-containing toothpaste and S-PRG filler-containing toothpaste. Similar amounts of fluoride ion were detected in the NaF-containing toothpaste specimen and S-PRG filler-containing toothpaste specimen. Strontium ion was detected only in the S-PRG filler-
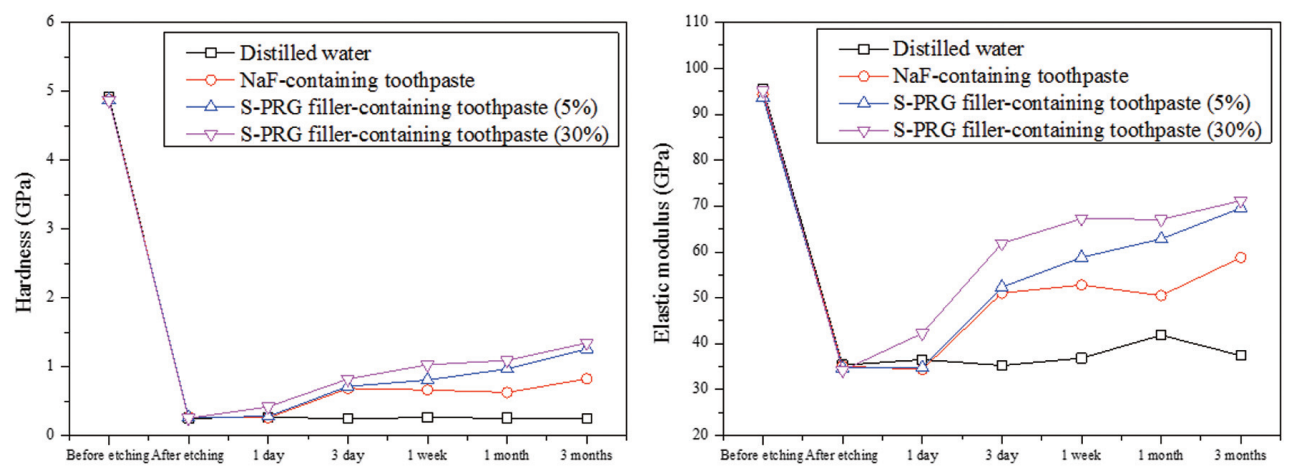

Fig. 2 Mean hardness and elastic modulus values of the buccal enamel surface before etching, after etching and during a 3-month immersion period.

Table 1 Mean values for hardness of the buccal enamel surface before etching, after etching and during 3 months of immersion periods $(\mathrm{GPa})$

\begin{tabular}{|c|c|c|c|c|c|c|c|c|c|}
\hline & \multicolumn{2}{|c|}{ Distilled Water } & \multicolumn{2}{|c|}{$\begin{array}{l}\text { NaF-containing } \\
\text { toothpaste }\end{array}$} & \multicolumn{2}{|c|}{$\begin{array}{l}\text { S-PRG filler- } \\
\text { containing } \\
\text { toothpaste }(5 \%)\end{array}$} & \multicolumn{2}{|c|}{$\begin{array}{c}\text { S-PRG filler- } \\
\text { containing } \\
\text { toothpaste }(30 \%)\end{array}$} & \multirow[t]{2}{*}{$\begin{array}{c}\text { 1-way ANOVA } \\
p \text { value }\end{array}$} \\
\hline & Mean & S.D. & Mean & S.D. & Mean & S.D. & Mean & S.D. & \\
\hline Before etching & 4.91 & 0.56 & 4.88 & 0.5 & 4.87 & 0.46 & 4.87 & 0.32 & 0.978 \\
\hline After etching & 0.25 & 0.09 & 0.27 & 0.15 & 0.26 & 0.09 & 0.25 & 0.08 & 0.895 \\
\hline 1 day & $0.26^{\mathrm{a}}$ & 0.09 & $0.26^{\mathrm{a}}$ & 0.1 & $0.28^{\mathrm{a}}$ & 0.15 & $0.42^{\mathrm{b}}$ & 0.18 & 0.0001 \\
\hline 3 days & $0.25^{\mathrm{a}}$ & 0.09 & $0.69^{b}$ & 0.31 & $0.71^{b}$ & 0.29 & $0.82^{b}$ & 0.22 & 0.0001 \\
\hline 1 week & $0.26^{\mathrm{a}}$ & 0.44 & $0.66^{\mathrm{b}}$ & 0.2 & $0.81^{b}$ & 0.33 & $1.03^{\mathrm{c}}$ & 0.34 & 0.0001 \\
\hline 1 month & $0.25^{\mathrm{a}}$ & 0.09 & $0.63^{b}$ & 0.21 & $0.97^{\mathrm{c}}$ & 0.34 & $1.09^{c}$ & 0.41 & 0.0001 \\
\hline 3 months & $0.25^{\mathrm{a}}$ & 0.07 & $0.83^{b}$ & 0.22 & $1.26^{\mathrm{c}}$ & 0.44 & $1.35^{\mathrm{c}}$ & 0.42 & 0.0001 \\
\hline
\end{tabular}

1-way ANOVA followed by the Tukey test. Identical letters indicate that mean values were not significantly different. 
Table 2 Mean values for elastic modulus of the buccal enamel surface before etching, after etching and during 3 months of immersion periods $(\mathrm{GPa})$

\begin{tabular}{|c|c|c|c|c|c|c|c|c|c|}
\hline & \multicolumn{2}{|c|}{ Distilled Water } & \multicolumn{2}{|c|}{$\begin{array}{l}\text { NaF-containing } \\
\text { toothpaste }\end{array}$} & \multicolumn{2}{|c|}{$\begin{array}{l}\text { S-PRG filler- } \\
\text { containing } \\
\text { toothpaste }(5 \%)\end{array}$} & \multicolumn{2}{|c|}{$\begin{array}{l}\text { S-PRG filler- } \\
\text { containing } \\
\text { toothpaste }(30 \%)\end{array}$} & \multirow[t]{2}{*}{$\begin{array}{c}\text { 1-way ANOVA } \\
p \text { value }\end{array}$} \\
\hline & Mean & S.D. & Mean & S.D. & Mean & S.D. & Mean & S.D. & \\
\hline Before etching & 95.5 & 6.68 & 94.25 & 5.6 & 93.65 & 5.52 & 95.11 & 5.33 & 0.535 \\
\hline After etching & 35.35 & 10.36 & 35.16 & 9.24 & 34.71 & 11.78 & 34.21 & 10.2 & 0.969 \\
\hline 1 day & $36.56^{\mathrm{a}, \mathrm{b}}$ & 8.7 & $34.4^{\mathrm{a}}$ & 8.8 & $34.85^{\mathrm{a}}$ & 9.92 & $42.29^{b}$ & 10.78 & 0.003 \\
\hline 3 days & $35.29^{\mathrm{a}}$ & 7.53 & $51.07^{\mathrm{b}}$ & 15.4 & $52.35^{\mathrm{b}}$ & 14.57 & $61.87^{\mathrm{c}}$ & 14.9 & 0.0001 \\
\hline 1 week & $36.86^{\mathrm{a}}$ & 25.3 & $52.81^{\mathrm{b}}$ & 11.6 & $58.83^{\mathrm{b}, \mathrm{c}}$ & 14.67 & $67.27^{\mathrm{c}}$ & 12.02 & 0.0001 \\
\hline 1 month & $41.89^{\mathrm{a}}$ & 10.95 & $50.51^{\mathrm{a}}$ & 11.18 & $62.85^{\mathrm{b}}$ & 13.24 & $67.09^{\mathrm{b}}$ & 20.19 & 0.0001 \\
\hline 3 months & $37.42^{\mathrm{a}}$ & 10.4 & $58.79^{\mathrm{b}}$ & 9.7 & $69.55^{\mathrm{c}}$ & 16.2 & $71.2^{\mathrm{c}}$ & 15.2 & 0.0001 \\
\hline
\end{tabular}

1-way ANOVA followed by the Tukey test. Identical letters indicate that mean values were not significantly different.
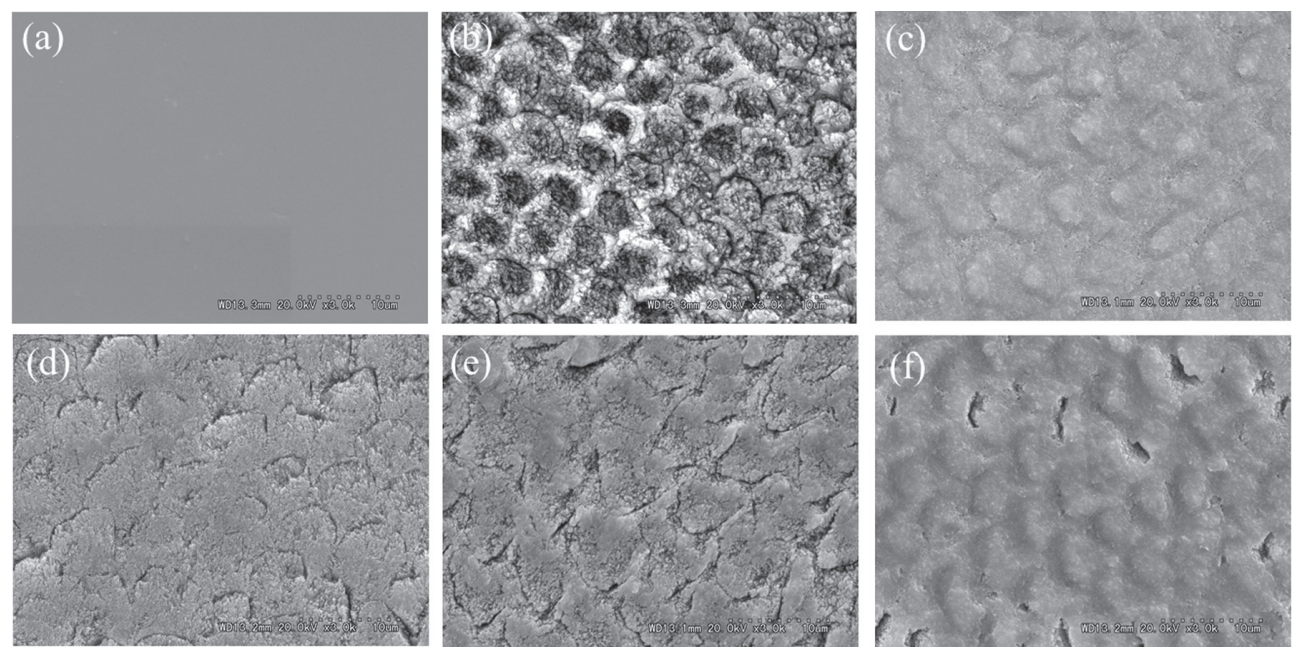

Fig. 3 SEM photomicrographs of (a) the original enamel surface, (b) an etched enamel surface, (c) a enamel surface after immersion in distilled water for 3 months, (d) a enamel surface after immersion in solution of NaF-containing toothpaste for 3 months, (e) and (f) enamel surface after immersion in solutions of S-PRG filler-containing experimental toothpastes [(e) $5 \%$; (f) $30 \%$ ] for 3 months.

Table 3 Composition of the enamel surfaces (wt. \%)*

\begin{tabular}{lcccccc}
\multicolumn{1}{c}{ Elements } & $\mathrm{Ca}$ & $\mathrm{P}$ & $\mathrm{F}$ & $\mathrm{Na}$ & $\mathrm{Sr}$ & $\mathrm{Al}$ \\
\hline Original enamel surface & 74.3 & 16.1 & 3.1 & 1.1 & 4.8 & 0.4 \\
Etched enamel surface & 70.3 & 19.2 & 5.6 & 1.4 & 2.8 & 0.6 \\
Distilled water & 76.4 & 12.4 & 4.3 & 1.3 & 4.8 & 0.8 \\
NaF-containing toothpaste & 73.1 & 17.2 & 5.5 & 1.5 & 2.2 & 0.6 \\
S-PRG filler-containing toothpaste (5\%) & 69.2 & 19.4 & 5.8 & 1.5 & 3 \\
S-PRG filler-containing toothpaste (30\%) & 67.9 & 20.8 & 5.5 & 1.3 & 3.3 \\
\hline
\end{tabular}

\footnotetext{
* Determined by EDS.
} 
Table 4 Mean amounts of released ions (ppm)

\begin{tabular}{lrrrrrr}
\hline \multicolumn{1}{c}{ Elements } & Al & B & Na & Si & Sr & F \\
\hline NaF-containing toothpaste & 0.1 & 0.6 & 555.6 & 42.0 & $-\quad 325.5$ \\
S-PRG filler-containing toothpaste (5\%) & 353.9 & 348.8 & 1259.2 & 7.1 & 824.2 & 383.0 \\
S-PRG filler-containing toothpaste (30\%) & 322.8 & 798.8 & 1489.0 & 13.4 & 1282.8 & 234.5 \\
\hline
\end{tabular}

containing toothpaste specimens. Greater amounts of $\mathrm{Al}, \mathrm{B}$ and $\mathrm{Na}$ ions were detected in the S-PRG fillercontaining toothpaste specimens compared to the NaFcontaining toothpaste specimen.

\section{DISCUSSION}

Etched enamel adjacent to brackets is susceptible to further demineralization. Additionally, because the enamel surface after bracket bonding using the conventional etch-and-rinse method has poor mechanical properties ${ }^{5)}$, the deteriorated enamel may lead to micro cracks within the enamel structure as a result of stress during orthodontic treatment and may also cause enamel fracture during debonding procedures ${ }^{2,19)}$. Also, pit-and-fissure sealant with resin-based materials requires an acid etching process. The acid etching destroys the enamel surface integrity, causing deepseated caries and reducing the caries-preventive effect of the sealant ${ }^{4}$. Thus, etched enamel after bracket bonding and sealant should ideally be remineralized as soon as possible. Because fluoride improves the acid resistance of enamel by acting on hydroxyapatite to convert it to fluoroapatite ${ }^{14,16,17)}$ and enhances mineral uptake during remineralization ${ }^{20)}$, fluoride-containing products, such as toothpaste, mouth rinse, and fluoridereleasing adhesives, have been used widely in clinical orthodontics $^{8-10)}$. The present in vitro study showed that the like-remineralization efficacy of S-PRG fillercontaining toothpaste for etched enamel was superior to that of NaF-containing toothpaste. Compared with the etched enamel surface, the mechanical properties of the specimens immersed in the solution of S-PRG filler-containing toothpaste after 3-month period were significantly enhanced by in vitro like-remineralization effects. However, the loss of hardness and elastic modulus of the enamel surface after acid etching was not totally recovered after the in vitro like-remineralizing behavior. The decreased hardness recovered by $24 \%$ and the elastic modulus by $61 \%$ after a 3 -month immersion in S-PRG filler-containing (30 wt\%) toothpaste; these values were higher than those for NaF-containing toothpaste (12\% for hardness, $40 \%$ for elastic modulus). According to the data for periods of less than 1 month, the solution of S-PRG filler-containing (30 wt\%) toothpaste showed earlier recovery of hardness and elastic modulus than the solution of NaF-containing toothpaste; this may be an advantage of S-PRG fillercontaining toothpaste. Thus, the null hypothesis (S-PRG filler-containing toothpaste would result in like-remineralizing effects equal to those of fluoridecontaining toothpaste) was partially rejected.

Although the mechanism of ion release from S-PRG filler is not completely understood, it was believed to have been due to the presence of a glass-ionomer phase around the glass core of the filler. In the present study, a considerable amount of ions, such as $\mathrm{Al}, \mathrm{B}, \mathrm{Na}, \mathrm{Si}$, $\mathrm{Sr}, \mathrm{F}$, were detected in the solution of S-PRG fillercontaining toothpaste; this is in agreement with a previous finding ${ }^{11)}$. These ions released from the S-PRG toothpaste may have contributed to the improvement of the mechanical properties of the etched enamel surface. However, the elemental analysis by EDS on the surface of the enamel after 3 months in vitro like-remineralization showed that similar amounts of calcium, phosphate, fluorine and strontium were detected in all specimens. A likely reason is that the depth from the enamel surface for like-remineralization behavior by released ions might be smaller than the detectable depth of the EDS analysis (approximately $1 \mu \mathrm{m}$ ). Further studies will be needed for depth-profiling using more surfacesensitive analytical methods such as X-ray photoelectron spectroscopy (XPS) and time-of-flight secondary ion mass spectrometer (TOF-SIMS). Si and $\mathrm{Al}$ are elements that form the structure of glass, while $\mathrm{Sr}$ and $\mathrm{F}$ are added into glass as a modifier. B plays both roles and is highly soluble. Regarding S-PRG filler-containing toothpaste with different concentrations, greater amounts of B, Na, Si and Sr ions were released from 30 wt\% S-PRG filler-containing toothpaste than from 5 wt\% S-PRG filler-containing toothpaste. However, the lower levels of $\mathrm{Al}$ and $\mathrm{F}$ ions were released from 30 wt\% S-PRG filler-containing toothpaste than from 5 wt\% S-PRG filler-containing toothpaste. A likely reason is that the elution rates with balance related to an equivalent amount of distilled water were different for each element. Strontiumapatite and fluoroapatite might have formed on the etched enamel surface by the released $\mathrm{Sr}$ and $\mathrm{F}$ ions. Because $\mathrm{Sr}$ is structurally similar to $\mathrm{Ca}$, it might have replaced the calcium released from the enamel surface ${ }^{21)}$. In the present study, a detectable amount of strontium was released from the enamel surfaces immersed in S-PRG fillercontaining toothpaste and this might confirm the hypothesis that strontiumapatite was formed on the etched enamel surface. Previous studies indicated that $\mathrm{Al}$ (aluminum lactate) is effective for dental hypersensitivity ${ }^{22}$ and B (boron-containing compound) has antibacterial and anti-inflammatory properties ${ }^{23)}$. The $\mathrm{Al}$ and $\mathrm{B}$ ions released from S-PRG particles 
in the present study might not contribute to likeremineralization behavior of enamel surfaces.

The enamel surface is often aprismatic and more highly mineralized than the enamel subsurface ${ }^{24}$. However, the enamel surface was removed completely by the polishing process in the present study to obtain flat and polished specimens in an attempt to standardize specimens for nanoindentation testing. Therefore, the mechanical properties of the polished (bulk) enamel might differ slightly from those of the top surface region. On the other hand, to investigate remineralization and demineralization of enamel and dentin in vitro, many recent studies used $\mathrm{pH}$-cycling experiments (demineralizing and remineralizing periods) to simulate the real oral environment. However, we used a remineralization-only model because it facilitated determination of the remineralization ability of S-PRG filler-containing toothpaste. The test protocol in the present study differs from in vivo conditions, and various methods have been used to assess deminerization and remineralization of teeth. It is difficult to compare our results with values in the literature, due to the multiple test configurations, assumptions and approximations integrated into the experimental methodologies ${ }^{25}$. Because we did not assess the effects of combinations of saliva and S-PRG filler-containing toothpaste on the remineralization of etched enamel surfaces, further research is needed to assess the effects of this combination. In addition, we used distilled water for immersion to investigate the like-remineralizing ability of NaF-containing toothpaste; thus the remineralization ability of $\mathrm{NaF}$ containing toothpaste could be improved using a $\mathrm{Ca}$ containing environment, such as artificial saliva.

After in vitro remineralization for 3 months, the honeycomb-like structure caused by acid etching was partially filled by deposited layers, which were thicker for specimens immersed in the solution of S-PRG fillercontaining (30 wt\%) toothpaste than those of other solutions. Although the specimens immersed in distilled water, the solution of the NaF-containing toothpaste, and the solution of S-PRG filler-containing (5 wt\%) toothpaste showed similarly thin deposited layers, their mechanical properties were different and there may be differences in the quality of the layers.

The use of microhardness measurements with a Knoop indenter has been a popular method for quantitatively investigating the demineralization of enamel $^{26,27)}$. Recent advances in the nanoindentation test allow the measurement of mechanical properties with extremely small volumes of material compared with the Knoop indenter. The hardness and elastic modulus can be determined simultaneously ${ }^{28-30)}$, and the elastic modulus of enamel is an important mechanical factor. Because enamel deteriorated by etching may develop cracks within its structure as a result of stress during orthodontic treatment and bracket debonding, nanoindentation testing for determining the fracture toughness should be investigated in future research to understand the changes in brittleness during demineralization and remineralization of the enamel structure.

From the findings of the present study, the daily application of toothpaste containing S-PRG may raise the $\mathrm{pH}$ in the surrounding environment, and inhibit demineralization of enamel, in addition to assisting remineralization of etched enamel. Furthermore, application of S-PRG toothpaste using a custom tray before sleep may also facilitate like-remineralization of the enamel surface. Further investigation is required to identify more effective application methods and specific protocols.

\section{CONCLUSIONS}

Under the conditions of this study, the following conclusions can be drawn:

1. The experimental S-PRG filler-containing toothpaste may enhance like-remineralizing ability of an etched enamel surface due to its ability to release various ions; the efficacy was found to be superior to that of $\mathrm{NaF}$ (950 ppm)containing toothpaste.

2. The mechanical properties of the specimens immersed in the solution of S-PRG fillercontaining toothpaste after a 3-month period were significantly enhanced due to the likeremineralizing ability, although the decreased hardness and elastic modulus of the enamel surface due to acid etching was not totally recovered.

\section{REFERENCES}

1) Buonocore MG. A simple method of increasing the adhesion of acrylic filling materials to enamel surface. J Dent Res 1955; 34: 849-850.

2) Newman GV. Epoxy adhesives for orthodontic attachments: progress report. Am J Orthod 1965; 51: 901-912.

3) Eliades T. Orthodontic materials research and applications: Part 1. Current status and projected future developments in bonding and adhesives. Am J Orthod Dentofacial Orthop 2006; 130: 445-451.

4) Shimazu K, Ogata K, Karibe H. Caries-preventive effect of fissure sealants containing surface reaction-type pre-reacted glass ionomer filler and bonded by self-etching primer. J Clin Pediatr Dent 2012; 36: 343-348.

5) Iijima M, Muguruma T, Brantley WA, Ito S, Yuasa T, Saito T, Mizoguchi I. Effect of bracket bonding on nanomechanical properties of enamel. Am J Orthod Dentofacial Orthop 2010; 138: 735-740.

6) Featherstone JD. Remineralization, the natural caries repair process-the need for new approaches. Adv Dent Res 2009; 21: 4-7.

7) Tschoppe P, Zandim DL, Martus P, Kielbassa AM, Enamel and dentin remineralization by nano-hydroxyapatite toothpaste. J Dent 2011; 39: 430-437.

8) Sudjalim TR, Woods MG, Manton DJ, Reynolds EC. Prevention of demineralization around orthodontic brackets in vitro. Am J Orthod Dentofacial Orthop 2007; 131: 705.e19.

9) Paschos E, Kleinschrodt $T$, Clementino-Luedemann $T$, Huth KC, Hickel R, Kunzelmann KH, Rudzki-Janson I. Effect of different bonding agents on prevention of enamel 
demineralization around orthodontic brackets. Am J Orthod Dentofacial Orthop 2009; 135: 603-612.

10) Kohda N, Iijima M, Brantley WA, Muguruma T, Yuasa T, Nakagaki S, Mizoguchi I. Effects of bonding materials on the mechanical properties of enamel around orthodontic brackets. Angle Orthod 2012; 82: 187-195.

11) Fujimoto $Y$, Iwasa M, Murayama R, Miyazaki M, Nagafuji A, Nakatsuka T. Detection of ions released from S-PRG fillers and their modulation effect. Dent Mater J 2010; 29: 392-397.

12) Ito S, Iijima M, Hashimoto M, Tsukamoto N, Mizoguchi I, Saito T. Effects of surface pre-reacted glass-ionomer fillers on mineral induction by phosphoprotein. J Dent 2011; 39: 7279.

13) Saito T, Toyooka H, Ito S, Crenshaw MA. In vitro study of remineralization of dentin: effect of ions on mineral induction by decalcified dentin matrix. Caries Res 2003; 37: 445-449.

14) Featherstone JDB, Shields CP, Khademazad B, Oldershaw MD. Acid reactivity of carbonated apatite with strontium and fluoride substitutions. J Dent Res 1983; 62: 1049-1053.

15) Dedhiya MG, Young F, Higuchi WI. Mechanism for the retardation of the acid dissolution rate of hydroxyapatite by strontium. J Dent Res 1973; 52: 1097-1109.

16) Iijima $Y$, Koulourides T. Fluoride incorporation into and retention in remineralized enamel. J Dent Res 1989; 68: 1289-1292.

17) Zero DT, Fu J, Espeland J, Featherstone JDB. Comparison of fluoride concentration in unstimulated whole saliva following the use of a fluoride dentifrice and fluoride rinse. J Dent Res 1988; 67: 1257-1262.

18) ISO 14577-1. Metallic materials - Instrumented indentation test for hardness and materials parameters- Part 1: Test method. 1st ed. International Organization for Standardization; 2002.

19) He LH, Fujisawa N, Swain MV. Elastic modulus and stressstrain response of human enamel by nano-indentation. Biomaterials 2006; 27: 4388-4398.

20) Cate JM, Arends J. Remineralization of artificial enamel lesions in vitro. Caries Res 1977; 11: 277-286.
21) Thuy TT, Nakagaki H, Kato K, Hung PA, Inukai J, Tsuboi S, Nakagaki H, Hirose MN, Igarashi S, Robinson C. Effect of strontium in combination with fluoride on enamel remineralization in vitro. Arch Oral Biol 2008; 53: 1017 . 1022.

22) Higuchi Y, Kurihara H, Nishimura F, Miyamoto M, Arai H, Nakagawa M, Murayama Y, Suido H, Tanii S. Clinical evaluation of a dental rinse containing aluminum lactate for treatment of dentinal hypersensitivity. J Clin Dent 1996; 7: 9-12.

23) Luan Q, Desta T, Chehab L, Sanders VJ, Plattner J, Graves DT. Inhibition of experimental periodontitis by a topical boron-based antimicrobial. J Dent Res 2008; 87: 148-152.

24) Poggio C, Lombardini M, Colombo M, Bianchi S. Impact of two toothpastes on repairing enamel erosion produced by a soft drink: An AFM in vitro study. J Dent 2010; 38: 868-874.

25) Eliades T, Bourauel C. Intraoral aging of orthodontic materials: the picture we miss and its clinical relevance. Am J Orthod Dentofacial Orthop 2005; 127: 403-412.

26) $\mathrm{Hu} \mathrm{W}$, Featherstone JD. Prevention of enamel demineralization: an in-vitro study using light-cured filled sealant. Am J Orthod Dentofacial Orthop 2005; 128: 592600.

27) Gorton J, Featherstone JD. In vivo inhibition of demineralization around orthodontic brackets. Am J Orthod Dentofacial Orthop 2003; 123: 10-14

28) Oliver WC, Pharr GM. An improved technique for determining hardness and elastic modulus using load and displacement sensing indentation experiments. J Mater Res 1992; 7: 15641583.

29) Rho JY, Pharr GM. Nanoindentation testing of bone. In: An YH, Draughn RA, editor. Mechanical Testing of Bone and the Bone-Implant Interface. Boca Raton: CRC Press; 1999. p. 257-269.

30) Iijima M, Muguruma T, Brantley WA, Mizoguchi I. Comparisons of nanoindentation, 3-point bending, and tension tests for orthodontic wires. Am J Orthod Dentofacial Orthop 2011; 140: 65-71. 